\title{
Polyvagal Neural Biofeedback Training by Pulse Regulated Abdominal Breathing in Sleep Disorder: A Suggestion for COVID-19 Patients
}

\author{
Mohammadjavad Hoseinpourfard ${ }^{1 *}$, Masoumeh Shajarian ${ }^{2}$ \\ ${ }^{1}$ Institute for Cognitive Science Studies, Shahid Beheshti University, Tehran, Iran \\ ${ }^{2}$ Tehran University of Medical Sciences, Tehran, Iran
}

*Corresponding Author: Mohammadjavad Hoseinpourfard, Ph.D. in Cognitive Neuroscience, Brain and Cognition, Institute for Cognitive Science Studies, Shahid Beheshti University, Tehran, Iran. Tel: +98-9370265689,

Email: hpf.javad@gmail.com

Received April 18, 2020; Accepted July 20, 2020; Online Published August 9, 2020

\begin{abstract}
Background: Nowadays, one of the most important social troubles is the sleep disorder that more than $40 \%$ of some population faced. Biofeedback (BFB) as a complementary method could reduce many of its effects.

Objectives: This study aims to show the effects of BFB on the promotion of sleep quality and control sleep disorder impacts.

Methods: An interventional study was conducted on 48 volunteer patients with sleep disorders. Twelve patients were included in each of four groups consisting of control. Pittsburgh Sleep Quality Index (PSQI) was used as a standard tool for sleep quality measurement in both groups. Abdominal breathing via chest breathing was trained as a BFB training exercise for patients. Heart rate variability (HRV) was measured before and after BFB training. Data were checked for outliers and normal distribution. SPSS version 22 and a diagram prepared by sigma plot version 14 did data analysis.

Results: The finding showed the promotion of sleep quality by pulse regulated abdominal breathing (PRAB) by a significant difference before and after BFB for four weeks.

Conclusion: Based on the finding of this study, polyvagal BFB training was utilized as a complementary method for HRV thus not only can it treat the patients with a sleep disorder but also promote sleep quality of the normal persons. Hence, the PRAB can be used for the patients with chief complain of sleep disorder.

Keywords: Sleep Disorder, Biofeedback, Heart Rate, Vagal Nerve, Neural Pathway, COVID-19
\end{abstract}

\section{Background}

Today, one of the most important social problems is the sleep disorder. It has become a global issue because more than $40 \%$ of the population have experienced it. ${ }^{1,2}$ Sleep disorder could occur through large-scale causes. Many of the cognitive features such as memory, attention, learning, decision-making, executive function, social behavior, etc. are affected by a sleep disorder. Meanwhile, it causes stress and anxiety. ${ }^{3-5}$

People worry about their healthy lifestyle and focus on nourishment, exercise, etc. They therefore try to have a healthy effective plan in their regime about these items. Many studies have shown that they need to add healthy sleep to these items. ${ }^{5-8}$

Various studies have indicated that patients doing physical exercise by non-pharmacological intervention improve autonomic dysfunction. ${ }^{9}$ They had many biological, psychological, social, and spiritual aspects of interaction. Changes in sleep and the mental health during this period enhance propensity to depressive symptoms, and create problems in physiological and psychosocial functioning. ${ }^{10}$ Trying to achieve a healthy lifestyle, we should pay more attention to a night of good sleep. Studies have shown a close relationship between stresses and sleep disorders. ${ }^{10}$ Many of psycho-physiologists use various types of interventions simultaneously with medical interventions to control sleep disorder. ${ }^{11}$ One of the recent intervention methods for a sleep disorder is the use of biofeedback (BFB) relaxation training such as heart rate variability (HRV) training and sleep quality. ${ }^{5,12,13}$ Abrahamic religions according to the necessary verdict try to approach a systematic verdict. ${ }^{14}$ For example, Islamic verdict command a lifestyle by following the sun. ${ }^{14,15}$

The relaxation can help patients to achieve a better HRV and control their stress and responses to stress factors. Today, we know that BFB is a powerful technique to reduce many types of disorders including the stress, and also it can lead to the promotion of the quality of life. ${ }^{6,13,16}$ Breathing

Copyright (C) 2020 The Author(s). This is an open-access article distributed under the terms of the Creative Commons Attribution License (http:// creativecommons.org/licenses/by/4.0), which permits unrestricted use, distribution, and reproduction in any medium, provided the original work is properly cited. 
techniques are part of traditional relaxation methods; however, their impact on psychophysiological variables related to sleep is still unclear. ${ }^{5,13,17}$

BFB techniques were developed by psychologists before year $1970 .{ }^{18}$ After then, BFB has a long history in which they have proved their therapeutic efficacy in a great variety of clinical disorders such as neuralgia, cardiovascular, gastrointestinal, chronic pain, dermatological, sleep, respiratory, trauma, stress, and many other disorders. ${ }^{19}$ Practical applications of the $\mathrm{BFB}$ include particular electroencephalographic BFB whose importance and clinical applications have grown and continued to grow rapidly. ${ }^{18}$

The BFB is a technique that comprised of two parts, "bio" and "feedback", the combination of which indicates the process of gaining greater mindfulness of many physiological functions in the human body. Some researchers define $\mathrm{BFB}$ as the process by which a person learns to constantly affect his physiological responses, exactly like when somebody make-up him/herself by a self-reporting. ${ }^{7,20}$

$\mathrm{BFB}$ includes the use of biological and electronic sensors that supervise human physiological reactions so that people can observe their body functions. BFB aims to understand these habitual patterns so that steps can be taken to change them to reduce the symptoms associated with various disorders and diseases. ${ }^{12,21,22} \mathrm{BFB}$ is a type of active conditioning, enabling the organism to control the physiological processes that are usually involuntary such as heartbeat by voluntarily utilizing various feedback including breathing., ${ }^{5,13}$

The Stress Eraser is a handheld BFB device that uses visual and auditory signals to train users to breathe in resonant frequency with individual heart rate. ${ }^{23}$ The impacts of BFB as an effective agent for HRV by pulse regulated abdominal breathing (PRAB) can improve sleep quality.

\section{Objectives}

The polyvagal BFB training as a complementary method for improving the HRV can not only treat the patients but also promote the sleep quality of normal individuals. Hence, the PRAB can use it for these patients with chief complaints of sleep disorder.

Here, we also reflect on our personal experience of meditation and healing practices, involving polyvagal and COVID-19, during the lockdown in Tehran. This experience involved the use of Global Coherence and Inner Balance apps to test the null hypothesis that healing meditations, as regular activities, expected to incur no significant change. ${ }^{24}$

The strategies discussed are not exclusively restricted to consideration during the COVID-19 pandemic. Most research studies before this period will continue to be a part of the biopsychological approach to endometriosis long after COVID-19 restrictions had been lifted. ${ }^{25}$

The COVID-19 pandemic was regarded as the heuristic field, for investigation and intervention, to complement other local and global initiatives in research, instruction counseling, and integral health promotion. ${ }^{26}$ In addition, neural BFB tools have shown promises to facilitate mental health self-management. ${ }^{27}$ Simple methods such as breathing exercises, $\mathrm{BFB}$, and mindfulness can be utilized to mitigate acute episodes of stress and anxiety, while BFB services can be useful for COVID-19 patients. ${ }^{28}$

\section{Methods}

During this interventional study, the scores of sleep quality were reported before and after the BFB training for all of the referred patients to a healthcare center at the northeastern of Tehran. Although the population for this study included all of the patients referred, the samples were selected randomly from volunteers of a Glen view clinic - as explained before a healthcare center at the northeastern of Tehran. Twelve patients in the four groups were randomly selected from the volunteers.

At the first visit, patients were given the required advice about the study and BFB training described for each of them. Then, they asked to sign a consent letter and complete the Pittsburgh Sleep Quality Index (PSQI) questionnaire. Patients were taken care of a normal diet plan to eat and also the instructions to perform the HRV training at home twice a day before and after night sleep and 15 minutes for each time. They had used this plan of HRV before and after sleep for none or bad sleep patients. Data analysis for distribution by Shapiro-Wilk normality test and the comparison for any significant difference between the scores before and after BFB by $t$ test has been done. The SPSS version 22 for the statistical conclusion and the sigma plot version 14 for designation the visual diagram have been utilized.

\subsection{Pittsburgh Sleep Quality Index}

To measure the level of sleep quality, the standard version of the PSQI is used. This self-report questionnaire assesses sleep quality for the 4 preceding weeks. Fourteen items were served to generate 7 component scores (whose values were between 0 and 3) as subjective sleep quality, sleep latency, sleep duration, habitual sleep efficiency, sleep disturbances, the use of sleeping medication, and daytime dysfunction. Measuring sleep quality by ranging from 0 to 42 is then calculated. The lower values indicated the state of better sleep quality. The score of 5 is considered as a borderline for sleep deprivation in similar studies. The total PSQI score was examined for reliability. A Cronbach's alpha coefficient for the total PSQI score was 0.79. This represented good internal reliability. ${ }^{29}$

\subsection{Pulse Regulated Abdominal Breathing}

The skills of abdominal breathing form a new breathing stereotype with a predominance of abdominal breathing over chest breathing. Patients trained by device montage include two sensors as respiration effort in abdominal and respiration effort in the thoracic area or chest wall. Data collection was done with recorded signals by the 
sensors that installed peculiarities. Respiratory sensors were attached to both of the abdomen and thoracic area assortment of stretch. The respiratory curves should have distinct inhalation and exhalation phases. Controlled indices were considered in 4 groups. Four groups include the control group consisting of the group 4-6 entitled with a rhythm of 4 pulses for inhalation and 6 pulses for exhalation, the group 6-9 entitled with a rhythm of 6 pulses for inhalation and 9 pulses for exhalation, and the last group 8-12 was entitled with a rhythm of 8 pulses for inhalation and 12 pulses for exhalation. The participants were trained in terms of breathing technique for 15 minutes before going to sleep and after the end of sleep. Then, the scores of sleep quality were compared by $t$ test after 28 days of PRAB practice at home.

\section{Results}

Forty-eight patients were trained for HRV BFB in the study. The mean age was $38.96 \pm 6.63$ years with gender distribution by $31 \%$ for males and $69 \%$ for females. PSQI score for patients that referred to the clinic was $6.27 \pm$ $0.26(n=24,95 \% \mathrm{CI})$, followed by HRV BFB practice after four weeks the average of the PSQI scores reached $5.3 \pm$ $1.03(\mathrm{n}=24,95 \% \mathrm{CI})$. PSQI score in the control group was $6.00 \pm 0.58$ before the PRAB and after that was $6.15 \pm 0.80$ $(\mathrm{n}=24,95 \% \mathrm{CI})$. PSQI score in the group 4-6 was $6.23 \pm$ 0.83 before the PRAB and after that was $5.62 \pm 0.65(n=24$, $95 \% \mathrm{CI})$. The score in the group of PRAB with 6-9 was $6.62 \pm 0.51$ before the PRAB and after that was $4.08 \pm 0.76$ $(\mathrm{n}=24,95 \% \mathrm{CI})$. Moreover, in the group of PRAB with 8-12 was $6.23 \pm 0.73$ before the PRAB and after that was $5.31 \pm$ $0.48(\mathrm{n}=24,95 \%$ CI) (Figure 1).

\section{Discussion}

Abdominal breathing showed a significant difference in sleep quality for both groups of PRAB. The score of 5 in PSQI is a critical point for judgment although many of the resources emphasized the other tools for measuring the quality of sleep..$^{10,22,30,31}$ Hence, we considered that PRAB by $6-9$ is an efficient method for meditation and quality of sleep in sleep disorder patients by maximum changes in HRV, too. Sasangohar et al in their study have pointed to mediation, sleep, and work-life balance, while achieving any kind of solutions for COVID-19, we need to buy time for effective antiviral medications and a vaccine for COVID-19. Meanwhile, there is a need to provide technical oversight to ensure that new designs meet minimum safety requirements. ${ }^{28}$

We cannot forget our responsibility in taking care of our self and caring for our grief. We are taking accountability of our actions, our emotional wellbeing, cognition, and sanity. Instituting a self-remedy in our home like practicing a simple technique of BFB is beneficial during this difficult time. Imagine yourself sitting or lying down on the sofa, watching your pulse in a quite ambiance manner. You feel your pulses rhythming each second and minute and as your pulses lower, you can feel the air entering into your lungs and your heart is beating slowly. Thinking of being able to control (and be in control) and bringing down your pulse is considered as mindful. ${ }^{32}$ In this way, we showed that cor pulmonale neural pathway BFB training could affect both the sleep quality and HRV.

The SHISO, a traditional term of respect for a human being, is used as an acronym for Spirit (Umoya), Heart (Inhlizyo), Image (Umcabango), Soul (Umphefumulo), and Oneness (Ubunye). The SHISO workshop takes the form of 5 steps, 1 for each letter of the acronym. ${ }^{26}$ Our findings in this study pointed to the HRV and focused not only on sleep quality but also on many other aspects of the human mind and body as a mental health factor and also a cognitive function although some studies reported no changes for both of them. ${ }^{5}$ Therefore, we believe that the PRAB could modulate the vagal nerve as an efficient agent including HRV, sleep disorder, temperature, and so on.

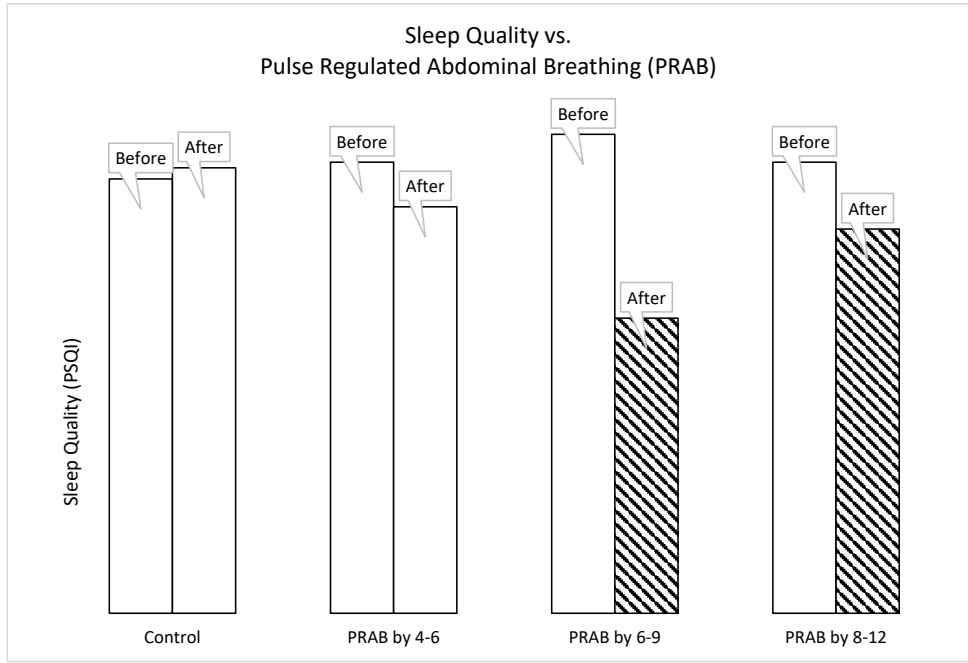

Figure 1. Comparison of PSQI Scores Before and After PRAB in 4 Groups Including Non-regulated, 4-6, 6-9, and 8-12. Hash signing was used for significant differences. This figure describes the sleep scores at the measured time points distributed in 4 groups including one group without PRAB, and 3 groups for PRAB by $4-6$ ( 4 pulses for inhalation and 6 pulses for exhalation), 6-9 (6 pulses for inhalation and 9 pulses for exhalation), 4-6 (8 pulses for inhalation and 12 pulses for exhalation). 
HRV BFB researcher focused on the age of cases and considered the adult populations with a variety of symptoms and signs. This study explores HRV BFB as a new treatment to improve cognitive functioning in older 31 compared to previous studies in which younger patients used HRV BFB training. ${ }^{10,11,13,20,31}$

\section{Conclusion}

Participants reported improved sleep quality and stress management. Neural BFB provides clarified knowledge about the specific biological activities that are available in neural aspects of behavior and their relations with the brain. When the brain receives information, it can use them to restore and reduce stress. The main aim of neural BFB is learning to pay attention to pulse regulated breathing as a neural mirror according to brain and functions. This study showed that neural BFB training can help patients to improve their psychophysiological mood and make better responses in stress and anxiety induced by sleep deprivation. ${ }^{27}$ Because of the above aspects, this study put forward a suggestion for patients with COVID-19.

\section{Authors' Contributions}

$\mathrm{MH}$ and MS contributed equally to this research.

\section{Conflict of Interest Disclosures}

The authors declare that they have no conflicts of interest.

\section{Ethical Approval}

This study has been registered in the Iranian Registry Clinical Trial (https://www.irct.ir/) with reference code: IRCT-41350.

\section{References}

1. Yang H, Sinicropi-Yao L, Chuzi S, et al. Residual sleep disturbance and risk of relapse during the continuation/ maintenance phase treatment of major depressive disorder with the selective serotonin reuptake inhibitor fluoxetine. Ann Gen Psychiatry. 2010;9:10. doi:10.1186/1744-859x-9-10.

2. Zarindast $M$, Nasehi $M$, Hoseinpourfard $M$. A mini review of serotonin and its receptors. Int J Med Rev. 2014;1(1):39-45.

3. Zwick GP. Neuropsychological assessment in autism spectrum disorder and related conditions. Dialogues Clin Neurosci. 2017;19(4):373-379.

4. Shereena EA, Gupta RK, Bennett CN, Sagar KJV, Rajeswaran J. EEG neurofeedback training in children with attention deficit/hyperactivity disorder: a cognitive and behavioral outcome study. Clin EEG Neurosci. 2019;50(4):242-255. doi:10.1177/1550059418813034.

5. Laborde S, Hosang T, Mosley E, Dosseville F. Influence of a 30-day slow-paced breathing intervention compared to social media use on subjective sleep quality and cardiac vagal activity. J Clin Med. 2019;8(2). doi:10.3390/jcm8020193.

6. Karimkhani S, Alizadeh Asli A, Namazi S, Yari M, Behrouz B. Relationship of sleep quality and sleep hygiene with islamic lifestyle and self-reported religious orientation in high school students. Journal of Research on Religion \& Health. 2018;4(3):7-20. [Persian].

7. Jester DJ, Rozek EK, McKelley RA. Heart rate variability biofeedback: implications for cognitive and psychiatric effects in older adults. Aging Ment Health. 2019;23(5):574-580. doi:1

\section{Research Highlights}

\section{What Is Already Known?}

The conditional normal technique in breathing enabled patients for better metabolism and body functions. On the other hand, we know also that sleep quality has a close relationship with many of the body functions. PSQI is one of the tools to measure this.

\section{What This Study Adds?}

In this study, breathing by specific discipline mentioned as PRAB affects internal and external attention states for all of the cases. Patients' sleep disorder is managed by this method. The most important complaints in patients with COVID-19 include stress, anxiety, and especially sleep disorder, which could be controlled by PRAB.

\subsection{0/13607863.2018.1432031.}

8. Rafati H, Tavakoli HR, Amerion A, Hoseinpourfard M, Nasiri T. Comparison of HACCP implementation requirements in nutrition department of two military and non-military healthtreatment centers. J Mil Med. 2010;11(4):191-196. [Persian].

9. Valenti VE, Kirizawa JM, Garner DM, Arab C. Considerations about heart rate variability in leukemia. Support Care Cancer. 2020;28(2):421-421. doi:10.1007/s00520-019-05197-y.

10. Kortesoja L, Vainikainen MP, Hotulainen R, et al. Bidirectional relationship of sleep with emotional and behavioral difficulties: a five-year follow-up of finnish adolescents. J Youth Adolesc. 2020;49(6):1277-1291. doi:10.1007/s10964-020-01203-3.

11. Yamazaki EM, Goel N. Genetics of circadian and sleep measures in adults: implications for sleep medicine. Curr Sleep Med Rep. 2020;6(1):32-45. doi:10.1007/s40675-020-00165-z.

12. Terroba-Chambi $C$, Bruno V, Vigo DE, Merello M. Heart rate variability and falls in Huntington's disease. Clin Auton Res. 2020. doi:10.1007/s10286-020-00669-2.

13. Lachapelle P, Cascon J, Pamidi S, Kimoff RJ. Accuracy of portable devices in sleep apnea using oximetry-derived heart rate increases as a surrogate arousal marker. Sleep Breath. 2019;23(2):483-492. doi:10.1007/s11325-018-1708-5.

14. Hoseinpourfard M, Ghanei M, Tofighi S, Ayoubian A, Izadi M. The Emergence of Hajj Stampedes: Lessons for Hajj Trauma Centers. Trauma Mon. 2017;22(4):e39455. doi:10.5812/ traumamon.39455.

15. Hoseinpourfard M, Ayoubian A. A review on some active websites about Islamic lifestyle based on webometric criteria [Persian]. Islamic Life Center Health. 2013;1(2):5-7. doi:10.5812/islamlifestyle.9010.

16. Aaraji F, Nosratabadi M, Hoseinpourfard M. The Impact of Biofeedback on Diabetic Patients' Glycemia. Hosp Pract Res. 2018;4(1):18-24. doi:10.15171/hpr.2019.03.

17. Brown BB. New Mind, New Body: Bio Feedback: New Directions for the Mind. Harper \& Row; 1974.

18. Carrobles JA. Bio/neurofeedback. Clin Salud. 2016;27(3):125131. doi:10.1016/j.clysa.2016.09.003.

19. Adam A, Claassen F, Coovadia A, et al. The South African guidelines on Enuresis-2017. Afr J Urol. 2018;24(1):1-13. doi:10.1016/j.afju.2017.07.002.

20. Sawyer C, Khayat RN. Role of wearable technology in the sleep-heart practice-a conceptual approach. Curr Sleep Med Rep. 2020;6(1):46-54. doi:10.1007/s40675-020-00167-x.

21. Sakakibara M, Kaneda M, Oikawa LO. Efficacy of paced breathing at the low-frequency peak on heart rate variability 
and baroreflex sensitivity. Appl Psychophysiol Biofeedback. 2020;45(1):31-37. doi:10.1007/s10484-019-09453-z.

22. Makarem N, Zuraikat FM, Aggarwal B, Jelic S, St-Onge MP. Variability in sleep patterns: an emerging risk factor for hypertension. Curr Hypertens Rep. 2020;22(2):19. doi:10.1007/ s11906-020-1025-9.

23. Tan G, Teo I, Srivastava D, et al. Improving access to care for women veterans suffering from chronic pain and depression associated with trauma. Pain Med. 2013;14(7):1010-1020. doi:10.1111/pme.12131.

24. Izadi M. Travel Medicine: A Country-to-Country Guide (Public Health in the 21st Century: Health Care Issues, Costs and Access). Nova Science Publishers, Inc; 2013.

25. Leonardi M, Horne AW, Vincent $K$, et al. Self-management strategies to consider to combat endometriosis symptoms during the COVID-19 pandemic. Hum Reprod Open. 2020;2020(2):hoaa028. doi:10.1093/hropen/hoaa028.

26. Edwards SD. AI in the noosphere: an alignment of scientific and wisdom traditions. Al Soc. 2020:1-3. doi:10.1007/s00146020-00999-9.

27. Hoseinpourfard M. Neural BioFeedback; As a "Psychophysiological Mirror": Protocol and Guidelines for
COVID-19 Patients for Stress, Anxiety, and Sleep Disorder. Saarbrücken, Germany: LAMBERT Academic Publishing; 2020. p. 276

28. Sasangohar F, Jones SL, Masud FN, Vahidy FS, Kash BA. Provider burnout and fatigue during the COVID-19 pandemic: lessons learned from a high-volume intensive care unit. Anesth Analg 2020;131(1):106-111. doi:10.1213/ane.0000000000004866.

29. Seaman C. An Evaluation of Fatigue Management Strategies Implemented on Hospital Nursing Units. Chapel Hill, NC: University of North Carolina at Chapel Hill Graduate School; 2015. doi:10.17615/e3cz-ey43.

30. Fox JL, Scanlan AT, Stanton R, Sargent C. Insufficient sleep in young athletes? causes, consequences, and potential treatments. Sports Med. 2020;50(3):461-470. doi:10.1007/ s40279-019-01220-8.

31. Mohit B, Wickwire EM. The health economics of sleep disorders among older adults. Current Sleep Medicine Reports. 2020;6(1):21-31. doi:10.1007/s40675-020-00166-y.

32. Sidi $\mathrm{H}$. The psychological sequelae during mental health and COVID-19 pandemic: learning from the past for today's coping styles. Med Health. 2020;15(1):1-4. doi:10.17576/ mh.2020.1501.01. 